Editor's Note: One of the big pluses associated with the recent International Congress on Microscopy Meeting in Durban, South Africa was the opportunity to visit with old friends. I've known Luc Harmsen, die Groot Baas at Anaspec South Africa, since the last Microscopy Society of Southern Africa's Capetown meeting sometime toward the end of the last century. Anaspec is a remarkable company that installs and services microscopes literally all over the world. They also put out a hilarious newsletter that Luc has been sending me since the Capetown meeting. Hilarious, yes, but jammed full of extremely useful material. The following is from Anaspec Info, Issue 36/02, October 2002, with Luc's permission. Check out www.anaspec.co.za for the golfing jokes in the referenced issue!

.. Ron Anderson, Editor

\section{Scan Speed, Mag and Accuracy. That is the Question!}

\author{
Luc Harmsen \\ Anaspec South Africa \\ luc@anaspec.co.za
}

In the past few weeks we have been very involved in setting up new QemSCANs, MLA's and analytical scanning systems and it is amazing how many times we had to explain the relationship between accuracy, magnification, scan speed, resolution and the resulting time taken to scan over a number of samples.

Let me explain. All the above systems are used to scan over a number of geological samples using a scanning electron microscope and EDX analytical systems. In most cases the users are simply looking for a phase/type of mineral within each sample. They then need to analyse it. The main criteria being that they scan through a large number of such samples in as short a time as possible.

Time being the critical problem here

The factors influencing this would be as follows:

* Magnification. If you run at a low magnification you end up scanning a large field of view and therefore would cover the sample in a few frames and so in theory get through the sample very quickly. The problem being that if you go very low in mag it takes a longer time to scan over that area and if you are looking for small particles, less than 10 microns, you may not see them. So low is good for speed but bad for accuracy. Scan speed. The faster you scan over the field of view the quicker you get through the field but again you loose on accuracy if you have smaller particles. On some SEMs you have a control called scan speed. In analytical systems this could be known as slew time or dwell time and then you select step size. This can be interpreted as the time taken for the e-beam to move from one pixel to the next, before the analysis is done. Then the step size would be the distance each pixel is from each other. On some ED system you select this by stating the resolution of the image.

* Image Resolution. On some SEMs scan speed may be known as resolution. This means how many lines in a frame. Too many and the field takes a long time, too little and you miss data.

Can you calculate the step size? Yes. Select a magnification and set up an image. Now use the SEM measuring annotation, that's if you have that option, and calculate the scanned area at that mag. Better known as the field width. For example field width at $150 \mathrm{x}$ mag on SEM X is $250 \mu \mathrm{m}$. If you now set a image resolution of $256 \times 256$ then each step is about $1 \mu \mathrm{m}$ apart because $256 \times 256$ means 256 pixels $\times 256$ pixels. 256 pixels into $250 \mu \mathrm{m}=\sim 1 \mu \mathrm{m}$ per pixel. If you select a lower resolution of $128 \times 128$ the scan will go faster but the step size will be about $2 \mu \mathrm{m}$ apart. If you are looking for $1 \mu \mathrm{m}$ particles you may miss them because the step size is too big.

Dwell time or slew time. How long or short should that be? As with anything in EM it all depends! If you move the beam too fast from pixel to pixel the image is unclear. If you move too slowly you may be damaging the specimen as you are putting too much energy into one spot for too long.

Spot size/ beam current. Now we need to analyse the image. For this we need to put in enough electrons to get back the correct information. This could be

— SE image. To get the best SE image, we would want a small spot size to give good resolution imaging. That is basic EM knowledge!

- BSE image. The back scatter image resolution is largely determined by the detector used, the sample and the KV of the SEM. Some BSE detectors have a better resolution at defining different "grey levels" than others. Then the combination of the energy of the beam or $\mathrm{KV}$ selected and the sample will give you an image that looks sharp or out of focus. This is due to the dew drop effect as demonstrated on the Monte Carlo software. Changing the spot size ( probe current, beam current, resolution setting depending on what type of SEM you have.) will only increase the number of electrons going in, and so coming back out, of the sample. This means that the BSE image will only appear less noisy with a bigger spot size.

- X-Ray acquisition. The bigger the spot size the more electrons into the sample the more the $\mathrm{x}$-rays out so the faster the count rate. Again the resolution is determined by the $\mathrm{KV}$ selection and the sample.

From this you will note that spot size is not that important in an analytical system. However if you have the spot size too big you may end up damaging the sample as it may not be able to handle the number of electrons at that energy.

* The sample to BSE detector and X-ray detector distance.

The closer the detectors are to the sample the lower the spot size to get a good collection of data. Both the BSE and EDX detectors rely on the "data" to enter the detector by chance. The closer the detector the higher the chance of the "data" entering the detectors. Fact!

* Filament setup. Then we get to the first step of setting up the scanning and that is the cathode/whenelt/gun set-up. By changing the filament saturation point, position in the cathode, cathode to anode distance and the bias, all the top conditions will change. In most cases the scanning is going to happen over a long period of time. In this time we need the beam to be as stable as possible. It would also be nice if the filament did not "pop" in that time.

Basically what we have learned over the past few months is that trying to set up a "system," any system, to do long runs, scanning samples for specific data takes a lot of trial and error testing with a good test sample. From this you will be able to set up different conditions for various samples depending on the data required.

Once this testing and trial period is over you should have a set of conditions which stipulates what mag and resolutions you would require to pick up certain particle sizes and what would be crucial in making sure you pick them all up.

\section{GOLFING JOKE from Anaspec Info:}

Golfer: "You've got to be the worst caddy in the world."

Caddy: "I don't think so sir. That would be too much of a coincidence." 


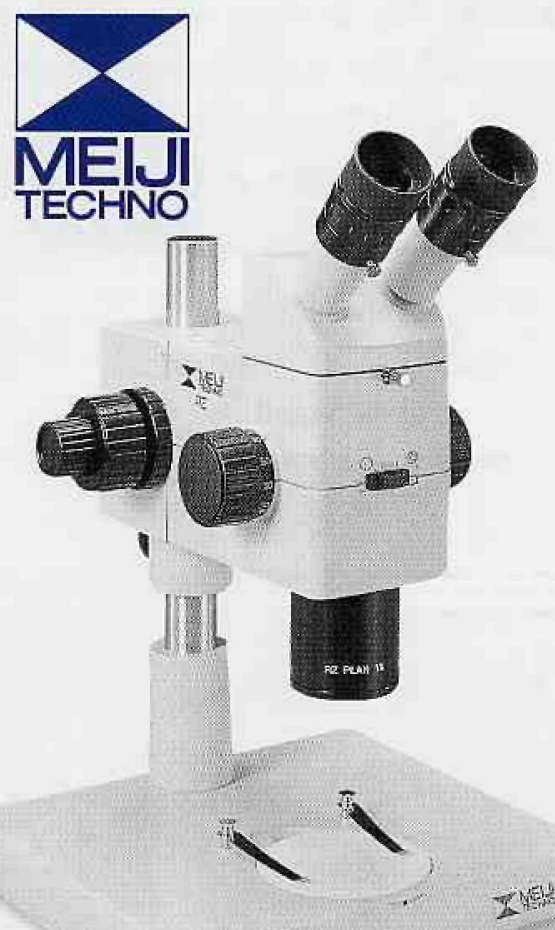

\section{The Meiji RZ Series of Research Stereo Microscopes.}

If you are looking for precision, durability, quality and value in a high performance Stereo Microscope, we invite you to take a closer look at Meiji's RZ Series of Research Stereo Microscopes.

The RZ Series modular system design allows you the freedom to
create an ideal instrument for your specific need or application.
Featuring a 10:1 zoom ratio, variable double iris diaphragm, and
positive detente click stops at 12 positions of magnification. A full
range of optional accessories is available, including: Video and
photo-micrographic systems, brightfield-darkfield transmitted light
stands, ergonomic binocular head, drawing attachment, multiple
interchangeable objectives and wide-field eyepieces. Complete
system versatility backed by a "Limited Lifetime Warranty."

For more information on these economically priced Stereo Microscopes, please call, FAX, write us or log on to our website today.

\section{MEIJI TECHNO AMERICA}

2186 Bering Drive, San Jose, CA 95131 ,

Tel: 408.428.9654, FAX: 408.428 .0472

Toll Free Telephone: 800.832 .0060 or visit our website at www.meijitechno.com



START by putting all of your instruments under one service contract with MAS (regardless of make or model). Our expert EM SERVICE GROUP has the knowledge and skills to keep your instrument working at its best.

\begin{tabular}{|cc|c|c|}
\hline \multicolumn{2}{|c|}{ TEM'S / SEM'S } & PREP EQUIPMENT & SPECIAL SERVIGES \\
\hline HITACHI & TOPCON & VACUUM COATERS & STAGES \\
JEOL & ISI & PLASMA ASHERS & BEAM BLANKERS \\
AMRAY CAMBRIDGE & SPUTTER COATERS & CUSTOM DEVICES \\
& ION MILLS &
\end{tabular}

NEW NEW NEW

Authorized service representatives for Gatan preparation equipment and Topcon TEM'S.
Contracts and On-Demand Emergency Service at Reasonable Rates from Factory Trained Specialists.

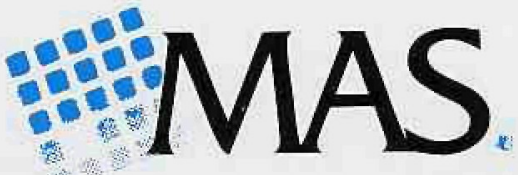




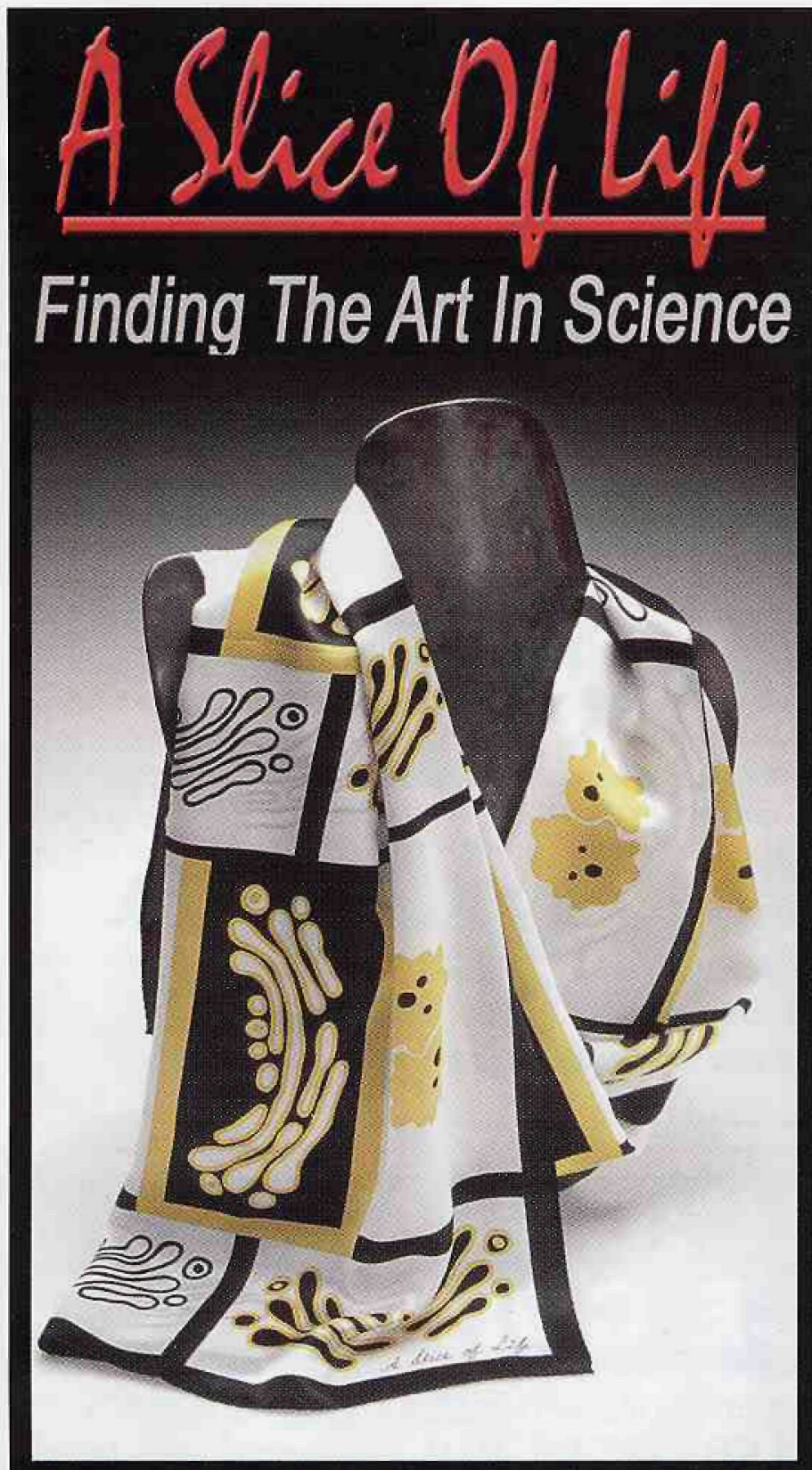

Experience amazing designs created by nature on elegant 100 percent silk scarves and ties. Patterns are derived from biological structures related to cell movement, energy production and performance, magnified 50,000 to $1,000,000$ times their original size.

For Information:



For only $\$ 3500$ for 5 days you could have a professional EM consultant provide SEM, TEM and EDS training, basic to advanced on site in your own laboratory on your own instruments, with consultancy too!

For more information

wWw. emcourses.com

or email

protrain@emcourses.com

\section{Microscopy Microanalysis}

Table of Contents Preview

Volume 9, Number 1, February 2003

Instrumentation and Technique

- Automated Analysis of SEM X-Ray Spectral Images: A Powerful New Microanalysis Tool

Paul G. Kotula, Michael R. Keenan, and Joseph R. Michael

A Simple Low-Vacuum Environmental Cell

Matthew H. Ervin

- A Single-Tilt TEM Stereomicroscopy Technique for Crystalline Materials

Rodney J. McCabe, Amit Misra, Terence E. Mitchell, and

Kathleen B. Alexander

Enhanced Compositional Contrast in Imaging of Nanoprecipitates Buried in a Defective Crystal Using a Conventional TEM Ute Kaiser and Andrey Chuvilin

Microanalysis

- Electron lonization Cross Sections for Atomic Subshells Peter Rez

Biological Applications

- The Role of Microscopy in Understanding Atherosclerotic Lysosomal Lipid Metabolism

W. Gray Jerome and Patricia G. Yancey

Apoptosis Process in Mouse Leydig Cells During Postnatal

Development

Maria José Salles Faria, Zilá Luz Paulino Simões, Laurelucia Orive Lunardi, and Klaus Hartfelder

Simultaneous Mechanical Loading and Confocal Reflection Microscopy for Three-Dimensional Microbiomechanical Analysis of Biomaterials and Tissue Constructs

Sherry L. Voytik-Harbin, Blayne A. Roeder, Jennifer E. Sturgis,

Klod Kokini, and J. Paul Robinson

Book Review

- Gold and Silver Staining: Techniques in Molecular Staining Morphology

Ken Tiekotter

News and Commentary

- Calendar of Meetings and Courses

Indexed in Chemical Abstracts, Current Contents, and BIOSIS

MSA members receive both Microscopy Today and Microscopy and Microanalysis FREE! 